\title{
La polyphonie discursive : un modèle ancré sur la ScaPoLine et le dialogisme
}

\author{
Francis Badiang Oloko (Université de Bergen, Norvège)
}

\begin{abstract}
The present work is intended as a contribution to the development of a discourse polyphony approach. Polyphony in language studies can take different meanings depending on the various approaches as Nølke, Fløttum, and Norén (2004) report. There has been a proliferation of perspectives and approaches to multivoicedness since the early works of Ducrot (1984). Ducrot himself was largely inspired by Genette's theses as well as by Bally's works. The link between him and Bakhtin's legacy is not as direct as it seems with literature (Nølke 2017). It is quite the opposite with dialogism theory (Bres 1999). The latter appears to draw more directly from Bakhtin (Dendale and Coltier 2006). Some of the approaches to multivoicedness are described as linguistic due to their focus on language features solely. Others on the other hand transcend the limits of language and integrate contextual information. Gjerstad (2011) is the first attempt to develop a model of polyphony whose focus is beyond mere linguistic features. His discourse polyphony approach takes into account intentionality and the interactive nature of speech. This was achieved through the combination of the ScaPoLine approach with dialogism and the Geneva Model. The present contribution bridges from Gjerstad's (2011). However, it limits the scope to the blending of the ScaPoLine and dialogism. After answering the question about the relevance of a discourse polyphony approach, this article offers a description of its scope, and the consequences of the incorporation of contextual elements into the polyphonic configuration taken up from the ScaPoLine. The result is tested on two concrete examples. If the conclusions to these examples approve the need for a discourse polyphony approach, its implementation requires some flexibility to match the dynamic nature of discourse also. Similarly, the relevance of context must be looked at with great caution.
\end{abstract}

Keywords: polyphony, ScaPoLine, dialogism, discourse polyphony, voices

\section{Introduction}

Le présent travail se veut une contribution à l'élaboration d'une polyphonie discursive. Le concept de polyphonie à lui seul dans les études de la langue peut prendre diverses acceptions selon les écoles et leurs orientations respectives. Nølke, Fløttum, et Norén (2004, 13) relèvent cette diversité des conceptions lorsqu'ils observent qu' " un bref aperçu - même rapide - de ses [la polyphonie du langage] différents emplois ou acceptions révèle vite que la polyphonie joue à plusieurs niveaux d'analyse et qu'il y a presque autant de conceptions de cette notion que d'auteurs qui s'en servent. » Depuis les travaux inauguraux de Ducrot (1984) sur l'élaboration d'une théorie polyphonique de l'énonciation, elle-même s'étant grandement 
inspirée des thèses de Bakhtine (1970), il y a eu un foisonnement des perspectives et des approches sur le phénomène de l'hétérogénéité énonciative. Cela ne doit pour autant pas être assimilé à un désordre mais bien plus à la richesse du phénomène en lui-même. On peut ainsi tout à fait observer que certaines de ces conceptions se réclament linguistiques par la priorité qu'elles accordent aux signes linguistiques, tandis que d'autres s'ouvrent à des considérations externes à la langue. Ces dernières, à l'instar de la praxématique, parlent donc davantage de dialogisme que de polyphonie dans ce cas. Gjerstad $(2011,73)$ souligne dans ce sens que

La ScaPoLine est une théorie sémantique, qui cherche à décrire la manière dont les voix font partie intégrante de la signification linguistique. Le dialogisme est d'ordre discursif, ce qui veut dire que la pluralité de voix est un phénomène susceptible de se produire dans l’interprétation, sans forcément relever du seul contenu sémantique de l'énoncé.

Le concept de polyphonie discursive est parmi les récents développements que l'on a enregistré dans le domaine de l'hétérogénéité énonciative. En effet, à travers ce concept, Gjerstad (2011) exprime son plaidoyer en vue d'un dialogisme ancré dans la langue et l'interaction. Bien plus qu'un simple plaidoyer, ses travaux sont un exemple de combinaison harmonieuse entre la polyphonie linguistique et le dialogisme. Sont donc pris en compte aussi bien les phénomènes linguistiques pures que des considérations qui ressortissent davantage des analyses interactionnelles. Les travaux de Gjerstad (2011) marquent ainsi le début d'une nouvelle acception de la polyphonie qui a ceci d'intéressant-entre autres spécificités de la conception - qu'elle veut analyser le matériau discursif dans ce qu'il a de particulier à savoir sa dualité constitutive en paramètres linguistiques aussi bien qu'en paramètres extralinguistiques (Maingueneau et Charaudeau 2002, 185-186).

De fait, la conception de la polyphonie discursive par Gjerstad (2011) est une combinaison de la ScaPoLine (Nølke, Fløttum, et Norén 2004), de la praxématique (Bres et Nowakowska 2006) et de l'école de Genève (Filliettaz et Roulet 2002). Sa perception s'ancre sur la ScaPoLine comme point de départ, pour s'ouvrir sur les deux autres théories qui lui permettent de prendre en compte des paramètres qui étaient jusque-là peu pertinents dans le champ d'application de la ScaPoLine. Il en va ainsi de l'intentionnalité qui est jugée inhérente à chaque discours. En effet, l'on peut convenir que dans un cadre normal, tout discours est précédé d'une intention communicative qui en influence la structure et le contenu ${ }^{1}$. Il est par conséquent pensable que le discours - pris comme résultat de l'énonciation - puisse porter les marques de cette intention même. Cela dit, Gjerstad $(2011,76)$ introduit cependant une nuance importante qui encadre l'application de l'intentionnalité du discours à l'analyse. Il estime en effet que

La polyphonie discursive est donc ancrée dans l'intentionnalité, mais c'est une intentionnalité qui ne doit pas être comprise comme celle de LOC, l'instance sémantique qui est le constructeur souverain de son énonciation. Car parlant de l'énonciation empirique, il ne faut pas oublier qu'elle est conditionnée par des mécanismes psychologiques qui échappent à la réflexion du sujet parlant lui-même. La mise en relation de l'intentionnalité et du dialogisme entraîne ainsi le risque d'attribuer au sujet parlant une maîtrise de la production du sens qui ne correspond pas à la réalité de la production verbale, laquelle se caractérise par le flou de la cognition.

\footnotetext{
${ }^{1}$ Nous nous limitons à ces deux tout en pensant que qu'il est possible que l'intention ait une influence sur bien d'autres paramètres mais cela relève d'une autre démonstration.
} 
Il convient de lire dans ce propos de Gjerstad une prudence qui permet de ne pas attribuer à tout va des intentions préalables à tout ce qui est exprimé. L'auteur cite les sciences cognitives qui peuvent démontrer un flou de la cognition qui est tel que l'on ne prémédite pas toujours ce que l'on dit, la maîtrise totale du dit étant elle-même relative.

La prise en compte des dimensions du dialogisme ${ }^{2}$ (Bres et Nowakowska 2006, 24-27) notamment permet, au cours de l'analyse, d'intégrer l'environnement du discours comme élément pertinent dans la construction du sens par celui-ci. Il faut donc croire que le discours se construit sur un fond - lexico-sémantique par exemple - élaboré par des discours précédents. Il est aussi une réponse à ces mêmes discours qui le précèdent, de même qu'il peut anticiper sur les autres discours qui lui répondraient.

Le modèle de Genève qui sert de troisième outil théorique à Gjerstad présente l'avantage d'avoir été développé dans l'optique d'analyser des productions verbales authentiques. De plus, elle subdivise la difficulté que présente l'analyse du discours en trois dimensions d'ordre linguistique, textuel et situationnel. Gjerstad $(2011,118)$ admet qu' " une telle subdivision permet de systématiser l'analyse d'un objet fort complexe, en explicitant l'interaction des facteurs qui occasionnent une interprétation donnée ».

La démarche de Gjerstad (2011) a une originalité théorique qui consiste en la combinaison de trois écoles de l'analyse énonciative dans le but de parvenir à une explication $\mathrm{du}$ phénomène polyphonique dans le discours. Elle présente aussi l'avantage d'utiliser le matériau linguistique comme point de départ tout en s'ouvrant à l'extralinguistique. Néanmoins il s'agit, pour chacune de ces trois approches, des objets théoriques fort complexes $^{3}$. Le résultat est un outil d'analyse qui va des paramètres linguistiques, vers l'intention communicative prise dans une perspective mesurée. Mais l'application de cet outil reste tout aussi complexe, voire élitiste car sujette à une maîtrise de tous les concepts opératoires que cela implique. Cette application dépend également des procédures qui, bien qu'héritées de la configuration polyphonique de la ScaPoLine, ne s'en retrouve pas simplifiée. C'est à ce niveau que réside encore l'enjeu de la présente contribution, c'est-à-dire reposer la problématique des contours d'une théorie polyphonique discursive. Quels sont donc les paramètres qui rentrent en jeu dans le schéma d'une polyphonie discursive simplifiée ? Mais avant d'y parvenir, il semble judicieux de revenir sur la question relative à l'utilité d'une polyphonie précisément discursive. La suite se consacrera au modèle précis que nous suggérons; celui-ci s'ancre dans la ScaPoLine et la praxématique essentiellement. Nous procèderons avec la complémentarité de la voix et du pdv dans une nouvelle configuration. L'ultime étape détaillera les contours d'une configuration discursive en présentant les êtres discursifs et en proposant deux exemples d'analyse. Nous conclurons par un bilan.

\section{Pourquoi une polyphonie discursive ?}

L'équation de la polyphonie a souvent trouvé dans de nombreux cas, une solution qui s'applique dans un domaine de définition délimité par le champ de la langue. En effet, Nølke

\footnotetext{
${ }^{2}$ Le dialogisme constitutif considère à la suite de Bakhtine que tout discours est constitué d'autres discours antérieur.

Le dialogisme interdiscursif saisit le discours dans sa rencontre avec des discours antérieurs dans sa saisie d'un objet du discours.

Le dialogisme interlocutif perçoit le discours dans son orientation vers le discours-réponse prévu.

${ }^{3}$ C'est le constat que nous faisons. Il ne s'agit pas d'une critique en soi ni de chacune de ces théorie, ni des conclusions de Gjerstad (2011).
} 
1994, 52 dans Dendale et Coltier $(2006,281)$ note qu'il y a " pour tout énoncé une sorte de contexte neutre qui est indiqué ou construit par la forme linguistique ». En se limitant donc à ce contexte dit neutre, on peut avoir un sens de l'énoncé. C'est l'esprit qui guide la polyphonie linguistique dont la ScaPoLine est l'une des illustrations les plus réussies ${ }^{4}$. Nølke, Fløttum, et Norén $(2004,15)$ notent dans le projet d'élaboration de cette théorie qu' «il devra s'agir d'une théorie qui pourra rendre compte des phénomènes polyphoniques proprement linguistiques, c'est-à-dire phénomènes relevant du système de la langue. » Cela est possible parce que « la langue, conçue comme le système linguistique à l'instar de Saussure, apporte des instructions relatives à l'interprétation polyphonique de la parole » (Nølke 2009, 82).

Pourtant, cette limitation du champ d'interprétation aux strictes instructions sémantiques que propose la langue pourrait s'avérer insuffisante lorsqu'on veut faire une analyse discursive, c'est-à-dire une analyse qui prenne en compte la nature du discours comme étant constitué aussi bien des paramètres linguistiques que des paramètres extralinguistiques (Maingueneau et Charaudeau 2002, 185-186). Une interprétation qui se limiterait à un seul membre de ce couple de paramètres pourrait ressembler à une « mutilation $^{5} »($ Gimbel 2016, 74) de l'objet d'analyse. Nølke $(2009,82)$ semble souscrire à cette posture quand il fait observer que

\begin{abstract}
On sait que le sens est toujours linguistiquement sous-déterminé : de multiples facteurs linguistiques et extralinguistiques concourent pour créer le sens, et par là les interprétations. Il y a donc tout lieu de penser que la polyphonie se crée par une combinaison, peut-être complexe, de phénomènes dont certains relèvent de la langue, d'autres de la (situation de) parole.
\end{abstract}

Cette position de Nølke est un aveu sans ambiguïté de la nécessité de transcender le niveau linguistique, sans pour autant le reléguer. Il est en effet légitime d'envisager comme cet auteur qu'en appliquant des paramètres extralinguistiques rigoureusement sélectionnés et selon des critères observables, on peut atteindre une interprétation qui n'est plus «sousdéterminée ». Cette démarche présentera en outre l'avantage d'exploiter le phénomène discursif dans sa dualité. Il ne s'agit pour autant pas de se servir de la langue comme d'un prétexte mais tout simplement comme le point de départ d'une analyse qui a tout simplement besoin de s'ouvrir à des paramètres nouveaux qu'offre le discours lui-même. Mais lesquels ? Gjerstad (2011) souligne les critères intentionnel et interactionnel du discours auxquels on pourrait ajouter le contexte, entendu ici comme la situation dans laquelle le discours se développe. Pourtant, il s'agit là d'une notion plutôt délicate.

«Le contexte d'un élément $X$ quelconque, c'est en principe tout ${ }^{6}$ ce qui entoure cet élément. » (Maingueneau et Charaudeau 2002, 134) Les auteurs observent que dans le cadre d'une unité linguistique, le contexte peut être l'environnement linguistique (ou verbal) de l'unité (cotexte) ou alors l'environnement non-linguistique. Ils soulignent en outre que le

\footnotetext{
${ }^{4}$ Tel est l'avis de Ducrot dans la préface de Nølke, Fløttum, et Norén (2004)

${ }^{5}$ Gimbel (2016) reprend l'argument de Dilthey qui reproche aux partisans d'une herméneutique objective (le positivisme) leur «obsession » pour l'objectivité qui mènerait à une mutilation de l'objet d'étude qui en science humaine n'a pas besoin d'être objectif. Nous reprenons simplement l'esprit de cette observation dans la prise en compte partielle de la nature de l'objet analysé qui pourrait mener à des résultats qui ne reflètent pas complètement l'objet en question.

${ }^{6}$ Il convient d'emblée de se démarquer de cette position en raison de son application très relative. On peut légitimement s'interroger sur la possibilité et même la nécessité de prendre en compte « tout » ce qui entoure une unité en vue de son analyse.
} 
contexte peut être envisagé de façon étroite ou alors de façon large. Ce qui pose une autre problématique, celle du point de vue selon lequel le contexte doit être envisagé. S'agit-il de celui de la production du discours ou celui de la réception. C'est en cela que la position de Schegloff 1997, 167 dans Micheli $(2006$, 5) nous semble intéressante. En effet, il dénonce le «theoretical imperialism » qui a souvent consisté pour les chercheurs à postuler les termes dans lesquels une interaction doit être analysée alors même que les interactants eux-mêmes établissent déjà au cours de l'interaction une grille de termes la rendant intelligible. On reste donc proche des thèses du contexte restreint pour ne pas se perdre dans des considérations qui sont bien au-delà du discours lui-même. L'effort en outre consistera pour la polyphonie discursive de limiter le contexte davantage au contexte de production et d'observer les éléments dans le discours qui sont pertinents pour sa propre analyse. Etant donné que le chercheur n'est pas toujours présent dans le contexte situationnel et temporel de développement du discours, une solution possible serait de n'émettre que des hypothèses qui ne seront définitives qu'après vérification.

La notion de contexte est essentielle en ceci que d'elle dépendent d'autres paramètres tels que les participants et les dimensions de dialogisme par exemple qui devront se limiter à un cadre strict. Il ne faut donc pas trop s'éloigner du texte mais réussir un savant dosage qui permettra - lorsque la nécessité se fait ressentir - de convoquer le contexte. Il ne s'agit par conséquent pas, dans la démarche qui est proposée ici de considérer le contexte comme un a priori mais bien plus de le différer et partir du contexte linguistique qui peut également suffire à rendre compte du sens d'une unité linguistique. C'est en procédant ainsi que nous nous rapprocherons de l'idéal que nous partageons avec Nølke $(2009,95)$

\begin{abstract}
Dès lors, le pas du discours idéalisé au discours authentique semble faisable. Il impliquerait le passage de l'ancrage linguistique à l'ancrage extralinguistique, à l'intermédiaire de la référence. Les ê-d deviendraient des images d'êtres en chair et en os. On ne serait plus dans le domaine de la linguistique de langue mais on franchirait la frontière de la pragmatique, de la sociolinguistique ou de la psycholinguistique - ou des études littéraires (!). Pourquoi ne pas oser faire ce pas ? Cela demanderait un développement méthodologique important, certes, mais tout porte à croire qu'un tel projet inter- ou transdisciplinaire porterait des fruits qui récompenseraient largement ce travail.
\end{abstract}

L'on peut retenir de cette étape que le développement d'une polyphonie discursive sert un besoin véritable, celui de prendre le discours dans sa nature afin d'en ressortir avec une interprétation qui sans vouloir être plus 'juste' que tout autre interprétation - le but n'est pas tant d'être plus juste qu'une autre - pourra tout au moins rendre au discours ce qui semble avoir été volontairement mis de côté et qui pourtant lui est inhérent. Nous n'excluons pas pour autant la quête d'un sens qui résulterait d'une combinaison du niveau linguistique avec les niveaux pragmatique et sociolinguistique - pour se limiter à deux des trois sphères d'application du discours relevées par Nølke. Cette ouverture clamée à un ancrage extralinguistique aura des incidences sur le schéma d'analyse. Il convient donc d'en préciser les contours afin d'en mesurer l'applicabilité par le billet d'exemples concrets.

\title{
3. Un modèle ancré sur la ScaPoLine et la praxématique
}

Ce modèle de polyphonie discursive revendique son héritage des développements faits par Gjerstad (2011). Comme son modèle donc, celui en cours de développement ici s'appuie en grande partie sur la ScaPoLine, théorie linguistique par excellence qui réclame entre autres 
son caractère structuraliste (Nølke, Fløttum, et Norén 2004, 28) lié au fait que la théorie " part d'une conception structuraliste de l'organisation de l'énoncé ». Mais à la différence de Gjerstad (2011), nous proposons de n'adjoindre à la théorie scandinave que la praxématique pour l'ouvrir à l'analyse proprement discursive. L'ambition simple de ce projet est rappelée par Badiang Oloko $(2017,43-44)$ : « This approach is expected to lead to a deeper and wellstructured exploitation of polyphony in discourse, in a bid to more clearly identify who is saying what and who is responsible for which position ». L'ambition n'est pas de de présenter de manière exhaustive ce qui fait la particularité de chacune de ces deux théories mais un point essentiel en ce qui concerne la ScaPoLine est son modèle d'analyse construit autour d'une configuration polyphonique; tandis que central à la praxématique est l'analyse des énoncés empiriques produits par des locuteurs empiriques, proches donc de l'existence réelle.

La configuration polyphonique est construite par le locuteur (Nølke, Fløttum, et Norén 2004, 30) mais Nølke (2008, 133) note que la création de cette configuration polyphonique « fait partie de l'interprétation que fait l'allocutaire du texte auquel il est confronté pris dans sa globalité ». En gros, la ScaPoLine estime que si c'est le locuteur qui construit la configuration, il appartient au chercheur de la retrouver et de la reconstituer schématiquement comme processus d'interprétation du texte. Il faut donc retenir que cette configuration est composée de quatre éléments constitutifs (Nølke, Fløttum, et Norén 2004, 30, Nølke 2008) :

- Le locuteur-en-tant-que-constructeur (LOC): il assume la responsabilité de l'énonciation ;

- Les points de vue (pdv) : entités sémantiques porteuses d'une source qui est dite avoir le pdv;

- Les êtres discursifs (ê-d) : entités sémantiques susceptibles de saturer les sources ;

- Les liens énonciatifs : ils relient les ê-d aux pdv. (Nølke 2008, 133)

Les points pertinents de cette configuration par rapport à ce projet sont, au-delà des points de vue qui émergent, le jugement mais surtout les rapports entre ces points de vue que la ScaPoLine appelle liens énonciatifs. Nous souscrivons alors à la position de Rabatel (2016, 14) qui rejoint ainsi les scapoliniens, à savoir que

Le but de l'analyse énonciative, lorsqu'elle est confrontée à un texte particulier, n'est pas de repérer des voix, des points de vue pour les étiqueter, au risque de les dissocier, mais de comprendre ce qui se joue dans la scénographie des voix et des points de vue, l'analyse énonciative gagnant à être prolongée par une appréhension herméneutique soucieuse d'un vrai et d'un bien communs.

Lorsqu'on applique donc ces préceptes, on obtient une structure de pdv en forme de $[\mathrm{X}]$ (JUGE (p)), où «X symbolise la source, JUGE le jugement et $p$ le contenu ». (Nølke 2008, 134)

L'apport de la praxématique porte pour l'essentiel sur la prise en compte des paramètres extralinguistiques dans la révision de cette configuration polyphonique. C'est donc le lieu souligner la notion essentielle du sujet parlant et donc de la voix qui acquièrent un caractère ubiquitaire puisque la condition sine qua non pour qu'il y ait un énoncé est la présence d'un sujet en amont doté de la compétence à en produire un. Bres et Nowakowska (2009, 105) s'inspirent d'une approche sémantique pour relever trois principales dimensions de la vocalité : (1) une dimension corporelle qui perçoit la voix en tant que caractéristique de 
l'être humain ; (2) une dimension discursive qui conçoit la voix comme un discours et (3) une dimension narratologique qui perçoit la voix en terme de sémiotique narrative. De ce triptyque, nous faisons le choix de ne conserver que la dimension discursive pour les simples raisons que l'on ne peut pas toujours vérifier que l'hétérogénéité énonciative soit à chaque fois liée à des entités physiques identifiables dans le monde réel. On conserve en effet la possibilité que le locuteur construise des 'voix' qui ne sont attribuables à aucune entité physique mais juste dans l'optique d'argumenter par exemple. Quant à la dimension narratologique, elle n'est pas pertinente dans les développements que nous envisageons. Nous considérerons donc la voix du point de vue du discours, autrement dit, tout discours représente au moins une voix. Cela entraine un empirisme relatif qui ne se lie pas trop à la réalité physique et humaine. Ceci implique par ailleurs que l'on définisse plus clairement les contours de l'application de ces termes et, comme on peut l'envisager, il sera nécessaire de réaménager aussi bien les éléments de la configuration que la terminologie à appliquer à ce modèle.

\section{Voix et point de vue : deux éléments complémentaires de la configuration}

Cette partie s'inspire essentiellement des travaux de Badiang Oloko (à paraître 2019). Il y est fait l'observation que la combinaison des voix et des pdv dans une nouvelle configuration destinée à l'analyse discursive aura pour, entre autres incidences, l'apparition des voix et des pdv. Néanmoins, ce à quoi réfèrent l'une et l'autre doit être précisé. Mais notons d'emblée qu'ils réfèrent à des manifestations différentes de l'hétérogénéité énonciative et pourtant complémentaires. Il cite ainsi Rabatel (2014, 186-187) qui, décrivant la voix, souligne qu'il s'agit de "l'instance première qui produit matériellement les énoncés, aussi cette instance est-elle affine à celle de voix, proférée (ou écrite) par un locuteur (ou scripteur) dotée d'une matérialité, subordonnée à l'expérience sensorielle. » Encore une fois, sans en arriver à l'expérience sensorielle, nous pouvons nous accorder avec Rabatel à croire que la voix est prise en charge par un locuteur et est responsable de l'énoncé tel qu'il apparaît. On en revient donc encore à sa nature ubiquitaire.

Le pdv quant à lui ne désigne plus une entité sémantique simplement comme le suggère la ScaPoLine. Il s'opère comme un retour à son sens originel, c'est-à-dire celui attesté dans la langue et qui est synonyme d'une opinion qu'une personne a à propos de quelque chose. Seront désignés comme pdv des instances de polyphonie déduites par inférence ou par présupposition. Autrement dit, on est tout à l'opposé de la voix qui se manifeste à travers une certaine matérialité discursive, c'est-à-dire, l'énoncé tel qu'il est rendu. On rejoint ainsi la conception de Perrin (2006, 29) qui estime qu'il existe des cas où l'expression est référentiellement opaque en raison du fait qu'elle ne réfère pas à ce qu'elle représente, mais à la façon dont quelqu'un voit, considère ce qu'elle présente, au sens de ses paroles ou pensées, auquel le locuteur réfère en le reproduisant mimétiquement dans son propre discours. Ce cas illustre ce que nous appellerons désormais pdv. 
On peut résumer les particularismes de ces deux concepts dans le tableau suivant :

\begin{tabular}{lcc} 
Critères & Voix & Pdv \\
\hline Paramètres non linguistiques & + & + \\
\hline Explicite & + & - \\
\hline Présupposé & - & + \\
\hline Inféré & - & + \\
\hline Référent empirique & + & $-/+$ \\
\hline Contenu exclusif & - & + \\
\hline
\end{tabular}

\subsection{Eléments non exhaustifs de comparaison entre 'voix' et 'pdv'}

Ce tableau démontre que l'on peut se servir de paramètres non linguistiques pour identifier l'une et l'autre. Mais la voix est plus explicite que le pdv (inféré ou présupposé). La voix ne peut être inférée et a un référent empirique identifié ou pas, critère qui est variable en ce qui concerne le pdv, c'est-à-dire qu'il peut avoir un référent empirique ou non. Le pdv peut exprimer tout juste un contenu tandis que la voix a besoin d'être concrète. Toutes ces différences seront matérialisées par des exemples concrets.

L'inclusion de la voix dans la configuration et la redéfinition de la notion de pdv sont les premiers points de démarquage par rapport à la configuration structuraliste de la ScaPoLine. Il faut sans doute souligner que ceci n'est en rien une critique formelle de ce schéma qui, dans l'analyse linguistique, a fait ses preuves et est apprécié au sein des études sur l'hétérogénéité énonciative. L'objectif est de la mettre au service d'un autre projet. Ce qui implique donc que ces aménagements iront bien au-delà des manifestations de l'hétérogénéité énonciative et atteindront la schématisation de ces manifestations.

\section{Une configuration discursive}

La configuration polyphonique que nous choisissons d'appliquer dans cette démarche discursive est inspirée essentiellement de celle de la ScaPoLine, de même que le modèle d'analyse. Elle se propose donc d'identifier la voix ou le pdv dans l'énoncé, d'en donner la source, ensuite le jugement et puis le contenu. Cela dit, cette disposition sera appliquée en rapport avec de nouveaux êtres discursifs, dans le but de satisfaire la nature discursive mais c'est aussi là que porte l'une des simplifications que nous proposons d'opérer.

\subsection{Les êtres en polyphonie discursive}

Les êtres discursifs sont une terminologie de la ScaPoLine (Nølke, Fløttum, et Norén 2004, Nølke 2008). Ils désignent des entité sémantiques - et par conséquent essentiellement linguistiques - qui saturent la source des pdv. Etant donné que la présente démarche est ouvertement discursive - mêlant le linguistique à l'extralinguistique, recourir à cette terminologie pour désigner des entités qui se situent justement en dehors de la langue pourrait 
trahir l'esprit des scapoliniens. En attendant de trouver une terminologie plus adaptée, appelons-les tout simplement des êtres.

Les êtres de la polyphonie discursive sont une reconfiguration de ceux de la ScaPoLine. Pour faire simple et aller directement au discours en tenant compte de son encrage empirique, Badiang Oloko (à paraître 2019) propose que soient retenus un nombre non exhaustif d'êtres en raison de leur fonctionnement dans le discours. Il s'agit du locuteur (L), du locuteur citant lui-même $\left(\mathrm{L}_{\mathrm{t}}\right)$, de l'allocutaire $(\mathrm{A})$, du tiers individuel $(\mathrm{T})$, du tiers individuel désigné ou nommé $\left(\mathrm{T}_{\mathrm{t}}\right)$ et du tiers collectif $(\mathrm{ON})$.

- le locuteur principal (L) : Il est la source de la voix, c'est-à-dire celui qui prend en charge la forme de l'énoncé tel qu'elle apparaît au lecteur. Le locuteur dont il est question ici est proche du locuteur axiomatique de la ScaPoLine (L-A) (Nølke 2008, 132). Mais contrairement à ce dernier qui construit des images de lui dans l'énoncé, le locuteur dont il s'agit ici est directement impliqué dans le discours en tant que responsable du dit. Il a donc un caractère ubiquitaire par son lien avec la voix qui est elle-même ubiquitaire en discours.

- le locuteur auto-cité $\left(\mathbf{L}_{\mathbf{t}}\right)$ : il s'agit des cas où le locuteur cite lui-même.

- l'allocutaire (A) : le terme désigne celui à qui s'adresse le locuteur. Il peut s'agir d'une personne unique ou alors d'un groupe de personnes à l'instar d'un public, une audience.

- le tiers individuel (T) : Il désigne un tiers individuel nommé, identifié ou désigné par le locuteur.

- le tiers inconnu $(\mathbf{X})$ : il désigne des cas où le locuteur ne sait pas ou alors ne donne pas nommément la source de l'autre voix/pdv. Il est ainsi des cas où des êtres sont justes créés dans le but de polémiquer de rendre plus claire sa propre position au cours d'un débat. Le dialogisme interlocutif de la praxématique peut parfois présenter ce cas de figure mais pas seulement ; la négation en est une autre illustration.

- le tiers collectif $(\mathbf{O N})$ : Il désigne un groupe d'individu plus ou moins clairement identifié. Il peut ainsi inclure L et/ou A selon l'orientation communicative de l'énoncé ; ou alors exclure l'un ou l'autre pour les mêmes raisons.

Il est important de souligner à la suite de ces êtres que la liste est non exhaustive. Il s'agit en effet d'êtres qui servent pour mettre en place l'approche discursive ici proposée. Ainsi, il est possible que d'autres êtres soient ajoutés à cette liste en fonction de leur pertinence dans la description du phénomène discursif. Il est en revanche tout aussi possible qu'ils évoluent en termes de leur contenu ou de leur référent. La nature du discours lui-même est foncièrement dynamique. Cela nécessite une certaine prudence lorsqu'on envisage un outil théorique qui vise sa description globale. Contrairement aux phénomènes linguistiques qui sont quasiment les mêmes pour tous - tout le monde puisant dans le même réservoir de règles et d'unités lexicales et sémantique par exemple - le phénomène discursif peut varier selon plusieurs critères : le locuteur et son statut, la thématique, les participants et leur statut, l'univers de croyance des uns et des autres, etc. Néanmoins, à partir de ces êtres et des descriptions précédentes, l'on peut proposer une analyse de certains phénomènes. 


\subsection{Analyse discursive}

Les prémisses qui précèdent permettent de proposer une analyse de quelques phénomènes. Pour montrer le passage de l'analyse linguistique (ScaPoLine) à l'analyse discursive, il semble propice d'adopter une démarche comparative. Elle permettra ainsi de faire ressortir à chaque fois ce qui est l'héritage de la ScaPoLine et ce que le schéma gagne grâce à l'apport discursif. Nous analyserons donc tour à tour la négation et la présupposition.

\subsubsection{Analyse discursive}

La négation est le marqueur polyphonique par excellence dans l'analyse linguistique notamment. Cela semble également être le cas dans l'analyse discursive. Il convient de mesurer le passage de l'une à l'autre et les implications que cela entraine. Soit donc le désormais fameux énoncé suivant :

(1) Ce mur n'est pas blanc?.

Le marqueur polyphonique mis en évidence est l'adverbe de négation $n^{\prime} \ldots$ pas. Il donne lieu à l'analyse suivante :

\section{ScaPoLine}

$\operatorname{Pdv}_{1}:[\mathrm{X}]$ (VRAI ('Ce mur est blanc'))

$\operatorname{Pdv}_{2}:\left[1_{0}\right]$ (INJUSTIFIÉ (pdv1))
Analyse discursive

$\operatorname{Pdv}_{1}:[\mathrm{X}]($ VRAI ('Ce mur est blanc'))

$\operatorname{Pdv}_{2}:[\mathrm{L}]\left(\right.$ INJUSTIFI $\left.\left(\mathrm{pdv}_{1}\right)\right)$

Voix : [L] VRAI ('Ce mur n'est pas blanc'))

L'analyse de la négation dans les deux tableaux précédents nous permet les commentaires suivants :

i. On passe de deux entrées à l'analyse linguistique à trois entrées à l'analyse discursive ;

ii. Il y a introduction de la voix qui prend en charge la forme et le contenu de l'énoncé tel qu'il est offert. Elle semble reprendre le contenu de $\mathrm{pdv}_{2}$ mais doit sa présence surtout à sa nature ubiquitaire.

iii. Le locuteur d'énoncé $\left(l_{0}\right)$ cède la place à un locuteur empirique $(\mathrm{L})$.

iv. Le tiers individuel inconnu $(\mathrm{X})$ est maintenu parce qu'aucune entité pouvant saturer la source de ce pdv n'est nommément désignée dans le cotexte. Et cet énoncé est analysé hors de tout contexte situationnel. Si cela avait été le cas, la source du pdv $\mathrm{p}_{1}$ aurait pu être (T).

v. On peut lire dans la configuration discursive, en outre, que l'on parle de $\mathrm{pdv}_{1}$ et $\mathrm{pdv}_{2}$ car dans l'un et l'autre cas, on procède par inférence. De plus, il faut croire qu'il s'agit davantage du contenu du $\operatorname{pdv}_{1}$ qui est «injustifié » par $\operatorname{pdv}_{2}$ plutôt que l'énoncé luimême.

\footnotetext{
${ }^{7}$ L'exemple est devenu classique mais nous le tirons de Nølke, Fløttum, et Norén $(2004,44)$.
} 
Du reste, on peut voir à travers l'analyse de la négation sur le plan discursif qu'il est possible d'harmoniser les pdv et la voix pour produire un résultat qui s'il se rapproche de l'empirique, ne se condamne pas toutefois à voir des êtres empiriques partout même lorsque cela ne semble pas être le cas. En plus, pdv $_{2}$ peut être décrit comme étant occasionné par l'énonciation. Cette analyse permettrait ainsi de retracer la chaîne énonciative qui mène à la négation telle que prise en charge dans la forme et le contenu de l'énoncé par le locuteur.

\subsubsection{La présupposition}

La présupposition est une instance de polyphonie qui admet deux pdv selon la configuration polyphonique de la ScaPoLine. L'analyse linguistique qui sera faite ici s'inspire de celle de Nølke (2008, 136). Soit l'énoncé suivant :

(2) Pierre a cessé de fumer ${ }^{8}$.

Le présupposé dans (2) réside dans la charge sémantique du verbe 'cesser'. L'auteur fait remarquer qu'il s'agit d'une analyse du présupposé aspectuel véhiculé par (2) comme un pdv dont ON assume la responsabilité. On obtient l'analyse qui suit.

ScaPoLine

$\operatorname{Pdv}_{1}:[\mathrm{ON}](\mathrm{VRAI}$ ('Pierre fumait autrefois'))

$\operatorname{Pdv}_{2}:\left[1_{0}\right]$ (VRAI ('Pierre ne fume pas actuellement'))
Analyse discursive

$\mathrm{Pdv}_{1}:\left[\mathrm{ON}_{\mathrm{L}+\mathrm{A}}\right](\mathrm{VRAI}$ ('Pierre fumait autrefois'))

$\mathrm{Pdv}_{2}$ : [L] (VRAI ('Pierre ne fume pas actuellement'))

Voix : [L] (VRAI ('Pierre a cessé de fumer’))

On peut faire les constats suivant par rapport à ces deux tableaux :

i. L'analyse discursive conserve les eux entrées de l'analyse linguistique. Avec le tiers collectif hétérogène 'ON' (Nølke 2008, 136) qui, dans les deux cas inclut aussi bien le locuteur que l'allocutaire, même si les référents de ces entités peuvent différer'. En effet, il est utile de rappeler que dans l'analyse linguistique il s'agit d'entités sémantique tandis que dans l'analyse discursive il s'agit de d'entités empiriques.

ii. Il y a introduction de la voix dans l'analyse discursive. C'est elle qui prend en charge le contenu véhiculé de l'énoncé, de même que sa forme.

Ces deux exemples n'épuisent certes pas le nombre, ni le type de transformations qui s'opèrent lorsqu'on passe de l'analyse scapolinienne pure à l'analyse discursive inspirée de la configuration de la ScaPoLine. Ils permettent néanmoins d'illustrer le type de changements susceptibles de se produire. On peut du moins retenir que la nature et le nombre de modifications à opérer dépendent de la nature du phénomène polyphonique analysé.

\footnotetext{
${ }^{8}$ L'exemple est de Nølke, Fløttum, et Norén $(2004,53)$ et de Nølke $(2008,136)$.

${ }^{9}$ Nous restons prudent à ce sujet car Nølke (2008) ne donne pas spécifiquement la composition de ON dans ce cas.
} 


\section{Bilan}

L'hétérogénéité énonciative est un phénomène complexe qui a vu émerger dans la recherche différentes positions qui permettent d'en rendre compte selon des optiques qui leur sont propres. Si d'aucuns font ainsi le choix de rester fidèle à la langue en menant des analyses essentiellement linguistiques (Nølke, Fløttum, et Norén 2004), d'autres s'ouvrent au non linguistique (Bres 1999, Nowakowska et Sarale 2011). Une autre voie plus récente choisit d'analyser le discours en lui restituant ses caractères extralinguistiques d'intentionnalité et d'interactivité (Gjerstad 2011). Notre contribution s'inscrit à la suite de Gjerstad (2011) et s'ancre sur la configuration de la ScaPoLine, tout en s'ouvrant à des données extralinguistiques (Bres et Nowakowska 2006) dans le but de proposer une analyse qui permette de restituer au discours ses caractéristiques linguistiques et extralinguistiques (Badiang Oloko 2017) et en vue de se rapprocher du référent du discours comme le souhaite Nølke (2009). Cela implique donc la perte de certains acquis de la ScaPoLine au profit de nouvelles considérations. Les changements orchestrés concernent principalement les êtres discursifs, le schéma d'analyse, l'intégration de la voix. Ces apports permettront en outre des interprétations qui sont plus proches de la réalité empirique et ont la caractéristique d'épouser la dynamique du discours. Il est donc possible que dans ce modèle que nous proposons, l'on ait à traiter des instances qui ne sont pas dites polyphoniques par défaut, mais que l'environnement discursif dans lequel ils apparaissent rend polyphoniques. C'est le cas de l'apposition par exemple (Badiang Oloko 2017, 46). Ce projet n'a pas la prétention de remettre en cause les évolutions plurielles dans le domaine de la polyphonie. Il veut apporter une plus-value à ce qui est déjà fait et dont il s'inspire. Il s'agit donc d'une perspective nouvelle qui, nous le pensons, si elle est bien structurée compte tenu de la complexité de la tâche que relève encore Nølke $(2009,95)$, peut porter satisfaction dans l'analyse du discours.

\section{Bibliographie}

Badiang Oloko, Francis. 2017. "Climate Change in Cameroon Political Discourse: A Case Study of Paul Biya's COP21 Speech." Bergen Language and Linguistics Studies 7. doi: 10.15845/bells.v7i0.1080.

Badiang Oloko, Francis. à paraître 2019. "Thèse de doctorat." Department of foreign languages, Université de Bergen.

Bakhtine, Michail. 1970. La poétique de Dostö̈evski. Paris: Seuil.

Bres, J., et A. Nowakowska. 2009. "Voix, point de vue... ou comment pêcher le dialogisme à la métaphore...." Cahiers De Praxématique 49:103-132.

Bres, Jacques. 1999. ""Vous les entendez ?" Analyse du discours et dialogisme." Modèles linguistiques, Association Modèles linguistiques/Editions des dauphins, 10 (2):7186.

Bres, Jacques, et Aleksandra Nowakowska. 2006. "Dialogisme: du principe à la matérialité discursive." In Le sens et ses voix: dialogisme et polyphonie en langue et en discours, edited by Laurent Perrin, 21-48. Metz: Université Paul Verlaine. 
Dendale, P., et D. Coltier. 2006. "Eléments de comparaison de trois théories linguistiques de la polyphonie et du dialogisme." In Le sens et ses voix: Dialogisme et polyphonie en langue et en discours, edited by Laurent Perrin, 271-299. Université de Metz.

Ducrot, Oswald 1984. "Esquisse d'une théorie polyphonique de l'énonciation." In Le dire et le dit, 171-233. Paris: Éd. de Minuit.

Filliettaz, Laurent, et Eddy Roulet. 2002. "The Geneva Model of Discourse Analysis: An Interactionist and Modular Approach to Discourse Organization." Discourse Studies: An Interdisciplinary Journal for the Study of Text and Talk 4 (3):369-393.

Gimbel, Edward W. 2016. "Interpretation and Objectivity: A Gadamerian Reevaluation of Max Weber's Social Science." Political Research Quarterly 69 (1):72-82.

Gjerstad, Øyvind. 2011. "La polyphonie discursive : pour un dialogisme ancré dans la langue et dans l'interaction." Université de Bergen.

Maingueneau, Dominique, et Patrick Charaudeau. 2002. Dictionnaire d'analyse du discours. Paris: Seuil.

Micheli, Raphaël. 2006 "Contexte et contextualisation en analyse du discours : regard sur les travaux de T. Van Dijk." SEMEN, Version mise en ligne le 28 avril 2007 (consultée le 28 septembre 2016) (21):1-17.

Nowakowska, Aleksandra, et Jean-Marc Sarale. 2011. "Le dialogisme : histoire, méthodologie et perspectives d'une notion fortement heuristique." Cahiers de Praxématique 57:9-20.

Nølke, Henning. 1994. Linguistique modulaire : de la forme au sens. Vol. 28, Bibliothèque de l'information grammaticale. Paris: Peeters.

Nølke, Henning. 2008. "La polyphonie linguistique avec un regard sur l'approche scandinave." Congrès Mondial de Linguistique Française, Paris.

Nølke, Henning. 2009. "Types d'êtres discursifs dans la ScaPoLine." Langue Française (164):81-96.

Nølke, Henning, Kjersti Fløttum, et Coco Norén. 2004. ScaPoLine : la théorie scandinave de la polyphonie linguistique. Paris: Kimé.

Perrin, L. . 2006. "Voix et points de vue dans le discours. De l'opacité linguistique à l'opacité référentielle des expressions " Le Français moderne 1 (74):22-31.

Rabatel, Alain. 2014. "Quelques remarques sur la théorie argumentative de la polyphonie." Arena Romanistica 21:185-204.

Rabatel, Alain. 2016. "L’Énonciation problématisante: En dialogue avec Le Royaume d'Emmanuel CarrèrePages." Arborescences: Revue d'Études Françaises 6:13-38. 